\title{
Head shape at age 36 months among children with and without a history of positional skull deformation
}

\author{
Brent R. Collett, PhD, ${ }^{1,2}$ Brian G. Leroux, PhD, ${ }^{3,4}$ Erin R. Wallace, PhD, ${ }^{2}$ Emily Gallagher, MD, MPH, ${ }^{5}$ \\ Jason Shao, BS, ${ }^{4}$ and Matthew L. Speltz, $\mathrm{PhD}^{1,2}$ \\ Departments of ${ }^{1}$ Psychiatry and Behavioral Sciences, ${ }^{3}$ Oral Health Sciences, ${ }^{4}$ Biostatistics, and ${ }^{5}$ Pediatrics, University \\ of Washington; and ${ }^{2}$ Center for Child Health, Behavior, and Development, Seattle Children's Research Institute, Seattle, \\ Washington
}

\begin{abstract}
OBJECTIVE In this study, the authors examined head shape through age 36 months for children with and without a history of positional plagiocephaly and/or brachycephaly (PPB).

METHODS Infants with PPB (cases) were identified through a craniofacial clinic at the time of diagnosis. Infants without diagnosed PPB were identified through a participant registry. Clinician ratings of 3D cranial images were used to confirm the presence or absence of PPB. The cohort included 235 case infants (diagnosed PPB, confirmed with 3D imaging), 167 unaffected controls (no diagnosed PPB, no deformation detected), and 70 affected controls (no diagnosed PPB, discernible skull deformation). Participants were seen in infancy (age 7 months, on average) and again at ages 18 and 36 months. At each visit, automated 3D measures of skull deformation quantified posterior flattening and generated an absolute asymmetry score. The authors also used automated 2D measures to approximate overall asymmetry (approximate oblique cranial length ratio) and calculate the cephalic index. They used linear regression to compare cases to unaffected controls and to compare affected versus unaffected controls on all measures. They also calculated the proportion of children in each group with "persistent PPB," defined as one or more head shape measures above the 95th percentile relative to unaffected controls at 36 months.
\end{abstract}

RESULTS Head shape became more rounded and symmetric for children with and without PPB, particularly between infancy and age 18 months. However, children with PPB continued to show greater skull deformation and asymmetry than unaffected controls at age 36 months. These differences were large in magnitude, ranging from 1 to 2 standard deviations (SDs), and in most (85.6\%) of the cases, there was evidence of persistent PPB at 36 months. Similarly, although differences were more modest (i.e., $0.26-0.94 \mathrm{SD}$ ), affected controls continued to exhibit skull deformation on most measures relative to unaffected controls and approximately $30 \%$ had persistent PPB. Within the case group, head shape at 36 months was similar for untreated patients with PPB and for those who received helmet treatment and for patients with and without a history of torticollis.

CONCLUSIONS Although head shape continues to improve, children with a history of skull deformation in infancy continue to exhibit measureable cranial flattening and asymmetry through age 36 months.

https://thejns.org/doi/abs/10.3171/2017.7.PEDS16693

KEY WORDS plagiocephaly; brachycephaly; head shape; deformation; infant; craniofacial

I NFANT positional skull deformation is characterized by posterior plagiocephaly (i.e., asymmetric head shape) and/or brachycephaly, which refers to a lower than normal ratio of skull length to skull width. ${ }^{4}$ Positional plagiocephaly and/or brachycephaly (PPB) has been common in the United States and other countries since the introduction of programs to prevent sudden infant death syndrome by encouraging supine sleep positioning for all infants.,13 Prevalence data are limited, but the best estimates suggest that $20 \%$ to $30 \%$ of infants show some degree of PPB. ${ }^{5,7,9}$

ABBREVIATIONS AAS = absolute asymmetry score; adj. diff. = adjusted difference; aOCLR = approximate oblique cranial length ratio; CI = confidence interval; LPFS = left PF score; PF = posterior flatness; PPB = positional plagiocephaly and/or brachycephaly; RPFS = right PF score; SD = standard deviation; SES = socioeconomic status. ACCOMPANYING EDITORIAL See pp 201-204. DOI: 10.3171/2017.8.PEDS17419.

SUBMITTED December 16, 2016. ACCEPTED July 26, 2017.

INCLUDE WHEN CITING Published online January 5, 2018; DOI: 10.3171/2017.7.PEDS16693. 
This has resulted in increasing numbers of children being referred to craniofacial centers and neurosurgery clinics for skull deformation and the growing popularity of associated treatments, including orthotic helmets and bands.

Little is known about the persistence of PPB beyond infancy, and most published studies have included children ages 24 months and under. Existing data suggest that the skull deformities associated with PPB improve with time regardless of treatment participation. For example, in a randomized trial, van Wijk et al. ${ }^{14}$ showed improvements in head shape for children assigned to receive helmet therapy and those who received only repositioning treatment. In one of the few studies to follow children beyond age 2 years, Hutchison et al. ${ }^{6}$ examined outcomes among children with a history of untreated PPB at ages $3-5$ years. They found improved head shape, and approximately $60 \%$ of the children who had PPB as infants were within the "normal" range on head shape indices.

We have followed since infancy a group of more than 200 children with a history of PPB (cases) and a smaller number of unaffected controls (167). The inclusion of a control group has allowed us to evaluate not only withincase-group change in head shape, but also the head shape of the children with PPB in relation to the presumably normal, but variable skull growth of typical children without calvarial deformation. In a previous study, we found that by age 18 months head shape became more rounded and symmetric among children with PPB and controls. ${ }^{3}$ However, regardless of their treatment history (i.e., orthotic helmet treatment vs no helmet treatment), children with PPB continued to show greater asymmetry and skull flattening than controls. In the current study, we sought to extend these findings in the same cohort to age 36 months. Specifically, we examined whether children with PPB would continue to differ from unaffected controls on automated 3D head shape measures. We also examined within-group changes over time, and potential modifiers of PPB, including history of orthotic helmet treatment, participation in physical or occupational therapy, and history of torticollis.

\section{Methods \\ Participants}

Prior to enrollment, we obtained informed consent for all participants using procedures approved by the institutional review board at Seattle Children's Hospital and in compliance with Health Insurance Portability and Accountability Act (HIPAA) standards.

\section{Infants With PPB}

Infants with PPB (cases) were recruited at the time of diagnosis in the Seattle Children's Hospital Craniofacial Center. Infants were eligible for participation in this group if they had been diagnosed with plagiocephaly or brachycephaly by a craniofacial specialist and were between the ages of 4 and 11 months. Exclusions were 1) history of prematurity ( $<35$ weeks gestation); 2 ) a diagnosed neurodevelopmental condition (e.g., Down syndrome), brain injury, or significant hearing or vision impairment; 3) presence of a major malformation or $\geq 3$ minor malformations; ${ }^{8}$ 4) diagnosis of craniofacial microsomia; 5) a non-English-speaking mother; 6) adoption or out-ofhome placement; and 7) family plans to move out of state before project completion. Between June 2006 and February 2009 , we recruited 235 infants with plagiocephaly or brachycephaly, representing $52 \%$ of all eligible case subjects. Participants were similar to nonparticipants with regard to demographic characteristics and severity of cranial deformation..$^{10}$

\section{Infants Without PPB}

The first 8 infants without PPB (controls) were recruited through area pediatric practices. The remaining controls were identified from a participant pool consisting of families in King and Snohomish Counties in Washington State who agreed to be contacted for research participation when their child was born. We contacted potential control families with a child in the target age range by phone and conducted a phone screening session with each family to determine eligibility. In addition to the exclusions listed for infants with PPB, infants without PPB were excluded if they had been diagnosed with PPB or any other craniofacial condition. We selected control participants who were similar to the case participants with regard to the distribution of the infants' age and sex and their family socioeconomic status (SES) and ethnicity. Two hundred thirtyseven control infants were recruited between June 2007 and February 2009, representing 90\% of those screened and determined eligible.

\section{Expert Ratings of Cranial Deformation}

After enrollment, infants' 3D cranial images were deidentified, randomly sorted, and rated by 2 dysmorphologists who were unaware of case status. Raters documented the degree of skull flattening, asymmetry, and brachycephaly using previously published 4-point severity scales (Cranial Technologies Inc. 2002 Rev 01). Raters then used this information to rate the overall severity of cranial deformation on a 4-point ordinal scale $(0=$ none, $1=$ mild, $2=$ moderate, $3=$ severe). Interrater agreement for case status (i.e., presence or absence of PPB using the overall severity of cranial deformation scale) was excellent $(\kappa=$ 0.80 ), and exact agreement for each of the 4 severity categories was good (weighted $\kappa=0.72$ ). The mean of the 2 raters' scores was used to represent the severity of each participant's cranial deformation. Infants with PPB were included in group comparisons if they had at least mild cranial deformation detected by one of the raters (i.e., average rating $\geq 0.5$ ), and controls were included if no plagiocephaly or brachycephaly was detected (i.e., all ratings $=0$ ). Control group participants without previously diagnosed deformation who had ratings indicating some degree of skull dysmorphology were retained in a separate group.

Based on these ratings, children were categorized into 3 groups: PPB cases (clinical diagnosis, confirmed with expert ratings), unaffected controls (no previous diagnosis, no evidence of skull dysmorphology with expert ratings), and affected controls (no previous diagnosis, but with detectable skull dysmorphology based on expert ratings of $3 \mathrm{D}$ images). 


\section{Study Procedures \\ 3D Imaging}

Children were first assessed in infancy (time 1). For participants with diagnosed PPB, the time 1 visit was scheduled within 4 weeks of diagnosis. Follow-up visits were scheduled with target ages of 18 months \pm 2 weeks (time 2) and 36 months \pm 2 weeks (time 3 ). 3D cranial images were obtained for all participants by using the 12-camera 3dMDcranial active stereo photogrammetry system.

\section{Medical and Intervention History}

Interviews were completed with mothers at time 1 to collect data on the child's medical history (e.g., history of torticollis) and demographic characteristics (e.g., family SES and ethnicity). At time 2 and time 3, abbreviated interviews were completed to document participants' treatment history, including orthotic helmet or band treatment and developmental interventions such as physical or occupational therapy. Mothers whose child received orthotic treatment (either a helmet or band) were asked, "How well do you think you followed through with suggestions for [child's name]'s helmet treatment for his/her plagiocephaly?" rated on a 5-point scale (very poor, poor, adequate, good, or very good).

\section{Image Analysis}

Procedures for generating automated head shape measures were described in detail by Atmosukarto et al. ${ }^{2}$ In brief, measures of posterior cranial flattening and asymmetry were derived by first constructing 2D histograms of azimuth-elevation angles of 3D surface normal vectors. Each histogram bin represented the percentage of surface normal vectors with a particular azimuth-elevation angle combination. Since the surface normal vectors of points that lie on a flat surface are almost parallel, they will have similar azimuth-elevation angles, leading to high-valued bins or peaks in the 2D histogram (Fig. 1).

\section{Posterior Flatness}

We used values from the bins representing similar azimuth-elevation angles to generate left and right posterior flatness scores (LPFS and RPFS, respectively). We

\section{Plagiocephalic head shape Brachycephalic head shape}

A
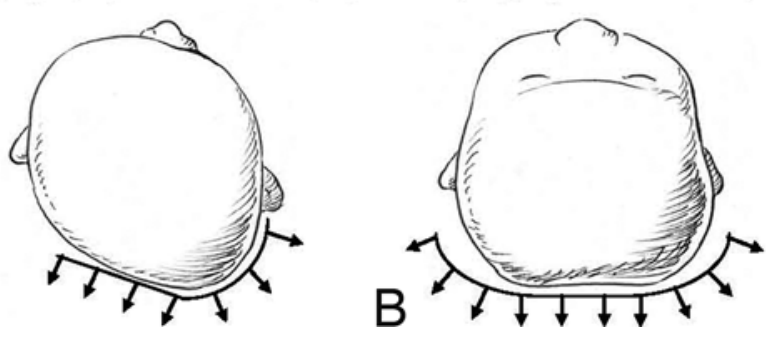

FIG. 1. Automated measures of posterior flattening for plagiocephalic (A) and brachycephalic (B) head shapes. For a child with a plagiocephalic head shape, surface normal vectors on the rounded surface have a wider distribution of angles than those on the flattened surface. This would be reflected in a higher PF score as well as a higher AAS. A child with a brachycephalic head shape would have a higher PF score, but minimal asymmetry. selected the greater of the LPFS and RPFS to determine the posterior flatness (PF) score.

\section{Posterior Asymmetry}

We generated an absolute asymmetry score (AAS), representing the absolute difference between the LPFS and RPFS.

\section{Skull Asymmetry}

We generated an approximate oblique cranial length ratio (aOCLR). We used a top-view 2D snapshot of the 3D head shape and measured the cross-diagonal length of the head contour to calculate the ratio of the longer to the shorter length.

\section{Brachycephaly}

We estimated the cephalic index by measuring the width of the head at the widest point divided by the length, using the same 2D snapshots used for the aOCLR.

To facilitate interpretation, we converted participants' scores to standardized z-scores. Separately for each head shape measure, we subtracted the mean for unaffected controls at time 1 from each participant's score and divided by the standard deviation (SD) for the unaffected controls at time 1 . By definition, this resulted in a mean of 0 and a standard deviation of 1 for unaffected controls on all measures at time 1 . We repeated this at time 2 and time 3, again standardizing each participant's scores using the group mean and standard deviations for unaffected controls at time 1. On all measures, higher scores reflect greater asymmetry or flattening. For example, a PF score of 2 would indicate that a child's PF score was 2 SDs greater than the average unaffected control score at time 1. All head shape measures are illustrated in Fig. 2.

\section{Data Analyses}

Descriptive statistics were performed to compare the diagnostic groups with respect to demographic characteristics. These analyses identified 5 outliers in the OCLR zscore distribution (all exceeding 10), which were excluded from subsequent analyses. Means and $95 \%$ confidence intervals (CIs) for z-scores were plotted by group over time. We used regression analyses to assess group differences, changes over time, and group $\times$ time interactions with adjustment for age at time 1, sex, race/ethnicity (white nonHispanic vs other), and SES (using a continuous variable ranging from 0 to 60 ). The analyses were performed using generalized estimating equations with robust standard errors to account for correlations among repeated observations involving the same participant. Finally, we established clinical cutoffs for head shape measures, using the 95th percentile based on time 3 control data for each measure (i.e., based on the distribution for affected and unaffected controls combined). We examined the proportion of children in each group who exhibited "persistent PPB," defined as a score above the 95th percentile on one or more head shape measures at time 3 .

\section{Results}

Time 3 data were available for one or more head shape 


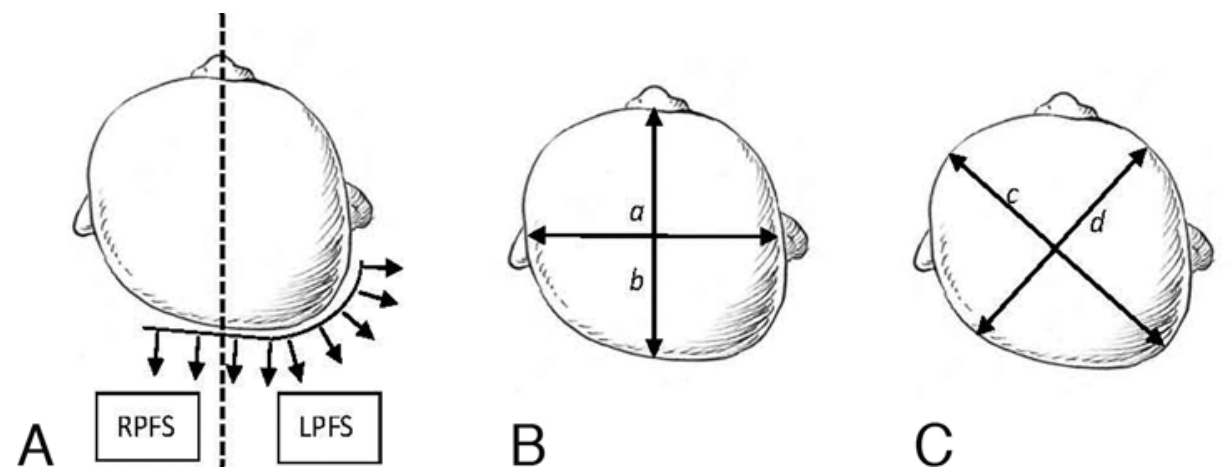

FIG. 2. Illustration of posterior flattening AAS, cephalic index, and aOCLR measures. A: The PF score reflects the number of similar azimuth-elevation angles on the posterior skull. The greater of the scores for the left and right sides (LPFS and RPFS) was used as the PF score. The AAS reflects the absolute difference in flattening on the left versus right side of the posterior skull (i.e., absolute differences between LPFS and RPFS). B: The cephalic index represents the width of the head at the widest point (a) divided by the length of the head $(b)$. C: The aOCLR is the ratio of cross-diagonal measures of head shape (c/d).

measures for 203 children with PPB, 63 affected controls, and 151 unaffected controls. This represents $86 \%$ to $90 \%$ of the original cohort. Complete data from all 3 assessments were available for $88 \%$ of the original sample. When compared with children lost to follow-up, retained participants were more likely to be white $(66 \%$ vs $42 \%$ for participants vs nonparticipants, respectively) and of higher SES (72\% of participants at SES categories of I or II vs $46 \%$ of nonparticipants). Nonparticipants and participants had similar severity scores for PF and aOCLR. However, nonparticipants had more severe posterior asymmetry and higher cephalic index scores at baseline than participants (average AAS 2.2 SD in nonparticipants vs 1.6 SD in participants; average cephalic index 2.0 SD in nonparticipants vs 1.4 SD in participants). Participant demographic and clinical characteristics are summarized in Table 1. Children in all groups were predominantly male, white and non-Hispanic, and of middle to upper SES. As expected, children with PPB were more likely to have a history of torticollis $(43 \%$ vs $3 \%$ in affected controls and $2 \%$ in unaffected controls) and to be a twin (11\% vs 3\% in affected controls and $0 \%$ in unaffected controls). Seventy-nine of the children with PPB (34\%) received orthotic helmet treatment, and compliance was described as "adequate" or better for 72 of these cases. One hundred thirteen $(50 \%)$ of the children with PPB received physical or occupational therapy, compared with 7 affected controls (10\%) and 6 unaffected controls (4\%).

\section{Group Differences at Time 3}

Children with PPB continued to show robust and statistically significant differences on all measures relative to unaffected controls (Fig. 3). After adjusting for age, sex, ethnicity, and SES, children with PPB had higher scores than unaffected controls on measures of posterior flatness (PF adjusted difference [adj. diff.] 1.10, 95\% CI 0.84-1.34) and posterior asymmetry (AAS adj. diff. 1.22, 95\% CI 0.94-1.50). They also had higher scores on measures of overall cranial asymmetry (aOCLR adj diff. $0.97,95 \%$ CI 0.79-1.15) and cranial width/length (cephalic index adj. diff. 1.94, 95\% CI 1.74-2.17). Similarly, affected controls had higher scores than infants without a history of skull deformation on most measures (adj. diff. 0.26-0.94); differences between affected versus unaffected controls were statistically significant with the exception of overall asymmetry (adj. diff. $0.26,95 \%$ CI -0.05 to 0.57 ).

In categorical analyses, $85.6 \%$ of cases $(n=172)$ had persistent PPB. The proportion of cases in which specific indices were above the 95th percentile ranged from $19.4 \%$ for AAS to $56.2 \%$ for aOCLR. By comparison, $30.2 \%$ of affected controls and $9.3 \%$ of unaffected controls had one or more scores above the 95 th percentile.

\section{Within-Group Changes in Head Shape and Potential Effect Modifiers}

In all 3 groups (i.e., cases, affected controls, unaffected controls), children's head shapes became more rounded and symmetric between infancy and age 36 months (Fig. 3). Changes in head shape were most pronounced in the children with $\mathrm{PPB}$, with reductions on all measures. Although much of this improvement (47\% to $82 \%$, depending on the measure) occurred between time 1 and time 2 (i.e., by approximately age 18 months), children with PPB continued to show modest improvements in head shape after 18 months. Affected controls showed declines in PF and cephalic index scores between time 1 and time 3 . Their asymmetry scores were relatively stable, with slight increases between time 2 and time 3 .

Within the PPB case group, children with a history of torticollis had similar scores to those without torticollis, though with slightly greater overall asymmetry (adj. diff. $0.47,98 \%$ CI $0.23-0.70$ ) and lower cephalic index scores (adj. diff. $-0.59,95 \%$ CI -0.92 to -0.26 ) (Fig. 4). Differences between children with PPB who received orthotic helmet treatment and untreated children with PPB were minimal for all time 3 measures (adj. diff. 0.07-0.21) (Fig. 5). After adjusting for PPB severity at time 1, change in head shape measures was similar for children with and without a history of helmet treatment (the larger observed reductions for treated cases from time 1 to time 3 could be explained by the fact that they started with higher baseline values). Similarly, only minor differences in time 3 measures were observed for children with PPB with and without a history of physical or occupational therapy (adj. diff. 
TABLE 1. Demographic and clinical characteristics by diagnosis group

\begin{tabular}{|c|c|c|c|}
\hline Characteristic & Unaffected Controls $(n=167)$ & Affected Controls $(n=70)$ & Cases $(n=233)$ \\
\hline \multicolumn{4}{|l|}{ Demographic characteristics } \\
\hline Mean age in mos at time $1, n=470$ & $6.9(1.7)$ & $6.7(1.6)$ & $7.2(1.6)$ \\
\hline Mean age in mos at time $2, n=457$ & $18.3(0.7)$ & $18.5(1.0)$ & $18.6(0.9)$ \\
\hline Mean age in mos at time $3, n=445$ & $36.0(1.1)$ & $35.9(0.9)$ & $36.5(1.2)$ \\
\hline \multicolumn{4}{|l|}{ Sex, $n=470$} \\
\hline Female & $74(44.3 \%)$ & $23(32.9 \%)$ & $80(34.3 \%)$ \\
\hline Male & $93(55.7 \%)$ & $47(67.1 \%)$ & $153(65.7 \%)$ \\
\hline \multicolumn{4}{|l|}{ Ethnicity, $n=470$} \\
\hline White (non-Hispanic) & $102(61.1 \%)$ & $43(61.4 \%)$ & $158(67.8 \%)$ \\
\hline Asian or Pacific Islander & $4(2.4 \%)$ & $9(12.9 \%)$ & $14(6.0 \%)$ \\
\hline Black & $5(3.0 \%)$ & $1(1.4 \%)$ & $0(0 \%)$ \\
\hline Hispanic or Latino & $22(13.2 \%)$ & $8(11.4 \%)$ & $28(12.0 \%)$ \\
\hline Native American & $0(0 \%)$ & $0(0 \%)$ & $0(0 \%)$ \\
\hline Mixed/other & $34(20.4 \%)$ & $9(12.9 \%)$ & $33(14.2 \%)$ \\
\hline \multicolumn{4}{|l|}{ Socioeconomic status, $n=470^{*}$} \\
\hline I (high) & $38(22.8 \%)$ & $23(32.9 \%)$ & $83(35.6 \%)$ \\
\hline II & $70(41.9 \%)$ & $30(42.9 \%)$ & $88(37.7 \%)$ \\
\hline III & $35(21.0 \%)$ & $14(20.0 \%)$ & $37(15.9 \%)$ \\
\hline IV & $19(11.4 \%)$ & $2(2.9 \%)$ & $18(7.7 \%)$ \\
\hline V (low) & $5(3.0 \%)$ & $1(1.4 \%)$ & $7(3.0 \%)$ \\
\hline \multicolumn{4}{|l|}{ Clinical characteristics } \\
\hline Torticollis, $n=470$ & $3(1.8 \%)$ & $2(2.9 \%)$ & $99(42.5 \%)$ \\
\hline Twin, $n=470$ & $0(0 \%)$ & $2(2.9 \%)$ & $27(11.6 \%)$ \\
\hline Received OT or PT by time $3, n=455 \dagger$ & $6(3.7 \%)$ & $5(7.4 \%)$ & $109(48.4 \%)$ \\
\hline
\end{tabular}

-0.37 to 0.30 ) (Fig. 6). These differences were attenuated after adjustment for baseline severity (adj. diff. -0.17 to 0.17). The proportion of children with persistent PPB was similar for children with and without a history of torticollis $(85.5 \%$ for children without torticollis, $87.0 \%$ for children with torticollis), for those with and without helmet treatment $(84.1 \%$ for children without orthotic treatment, $89.9 \%$ for children with orthotic treatment), and for those with and without physical or occupational therapy $(87.9 \%$ for children without physical or occupational therapy, $84.9 \%$ for those with physical or occupational therapy).

\section{Discussion}

This is the largest prospective study of head shape outcomes in children with PPB versus controls through age 36 months. Additionally, our study included children without a diagnosis of PPB who showed evidence of skull deformation in infancy. As observed in studies of this cohort at 18 months, ${ }^{9}$ head shape became more rounded and symmetric for all children by age 36 months. Changes in head shape were greatest in children with PPB and for affected controls, reflecting greater deformation in these groups at time 1. As part of their care in a craniofacial clinic, parents of children with PPB routinely received guidance in positioning practices prior to their child's enrollment in our study (e.g., increasing "tummy time" and other strategies for varying positioning of their baby). Further, a large proportion of children with PPB received physical or occupational therapy, which would often include parent guidance and other interventions to improve mobility. These "conservative management" strategies may have contributed to greater improvements in head shape over time relative to no treatment. Nonetheless, on average, children with PPB continued to show differences relative to unaffected controls on all head shape measures. These differences were large in magnitude (i.e., adjusted differences ranged from approximately 1 to 2 SDs) and statistically significant for all 4 shape measures. In approximately $86 \%$ of PPB cases, the children had persistent PPB, defined as one or more head shape scores above the 95th percentile relative to unaffected controls at time 3 . This percentage was similar for children with and without histories of orthotic helmet treatment, physical or occupational therapy, and torticollis. Affected controls also continued to show greater asymmetry, flattening, and brachycephaly than unaffected controls, although these differences were modest, and only $30 \%$ showed persistent skull deformation. 

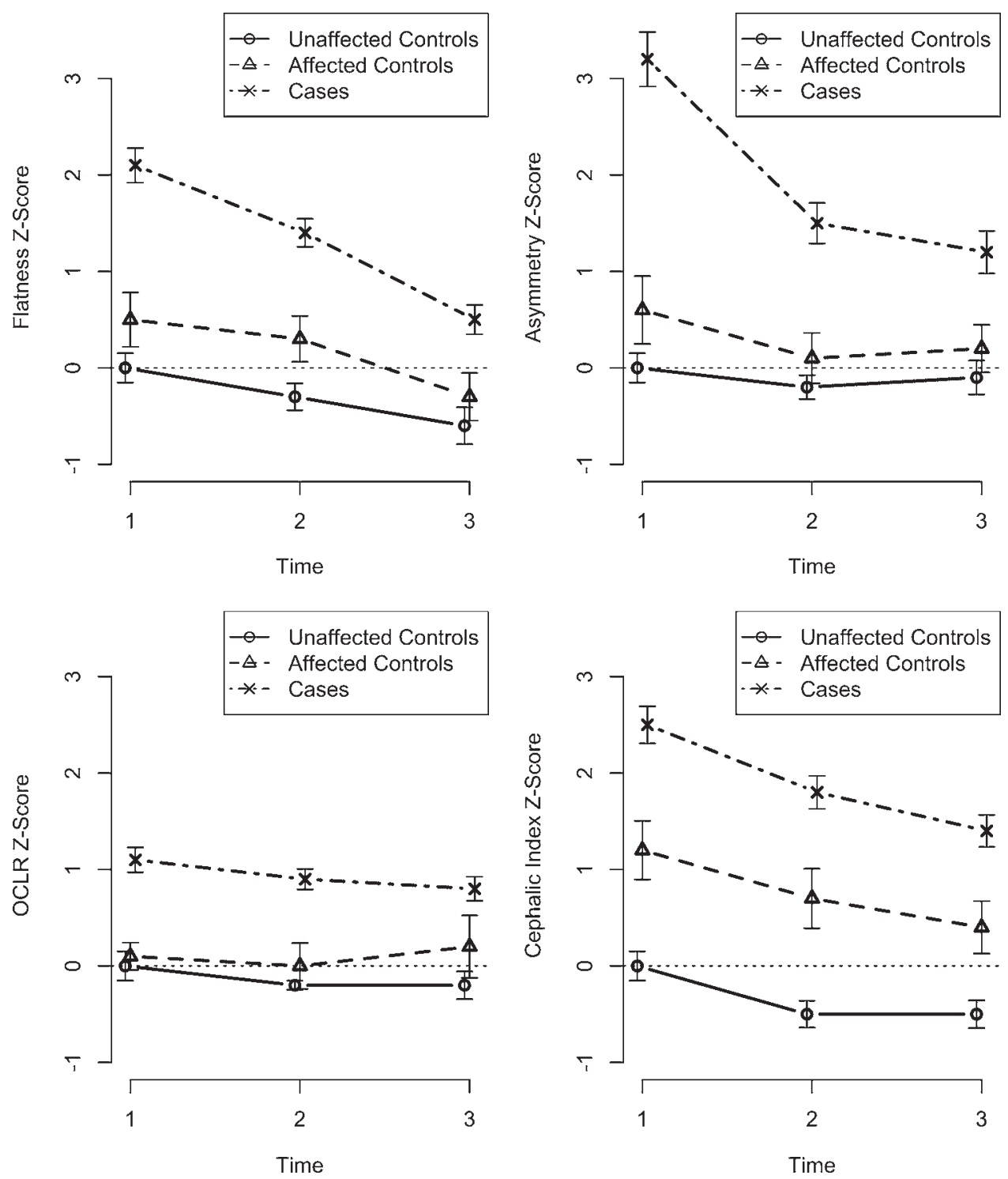

FIG. 3. Unadjusted mean values and $95 \%$ confidence intervals of head shape measures over time by diagnosis group. Head shape data were available for one or more measures for 167 unaffected controls, 70 affected controls, and 235 cases at time 1. At time 2, one or more measures were available for 161 unaffected controls, 68 affected controls, and 223 cases. At time 3, data were available for one or more measures for 151 unaffected controls, 63 affected controls, and 204 cases.

These findings are partially but not entirely consistent with previous research. Hutchison et al. ${ }^{6}$ found that $60 \%$ of the untreated children in their sample with PPB as infants were below a clinical threshold by age 4 years. More recently, Steinberg et al. ${ }^{11}$ found that deformation resolved by age 18 months in $89 \%$ of children with PPB who received only repositioning. Although we also found improvement in head shape through age 36 months, the vast majority $(85.6 \%)$ of children who had been diagnosed with PPB had persistent plagiocephaly, and there were large differences between children diagnosed with PPB in infancy and unaffected controls at this age. We found that most of the improvement in head shape occurred by 18 months. This was anticipated, given the rapid brain growth that takes place during a child's first 2 years of life, which helps to "round out" the skull. However, we also observed some continued reduction in flattening and asymmetry between 18 and 36 months in the PPB cases.

Among patients with PPB, those with a history of torticollis showed lower cephalic index scores and slightly higher scores on asymmetry measures. We found minimal differences in time 3 head shape measures as a function of orthotic helmet treatment and, after adjusting for initial PPB severity, found only modest differences in change over time between treated and untreated cases. The findings of previous studies are mixed with regard to the relative benefits of orthotic helmet therapy in relation to conservative management (i.e., repositioning and/or physical or occupational therapy) or the complete absence of treatment. Improvements in head shape following helmet therapy have been shown in several previous studies. ${ }^{15}$ However, the only randomized trial comparing helmet therapy to reposition- 

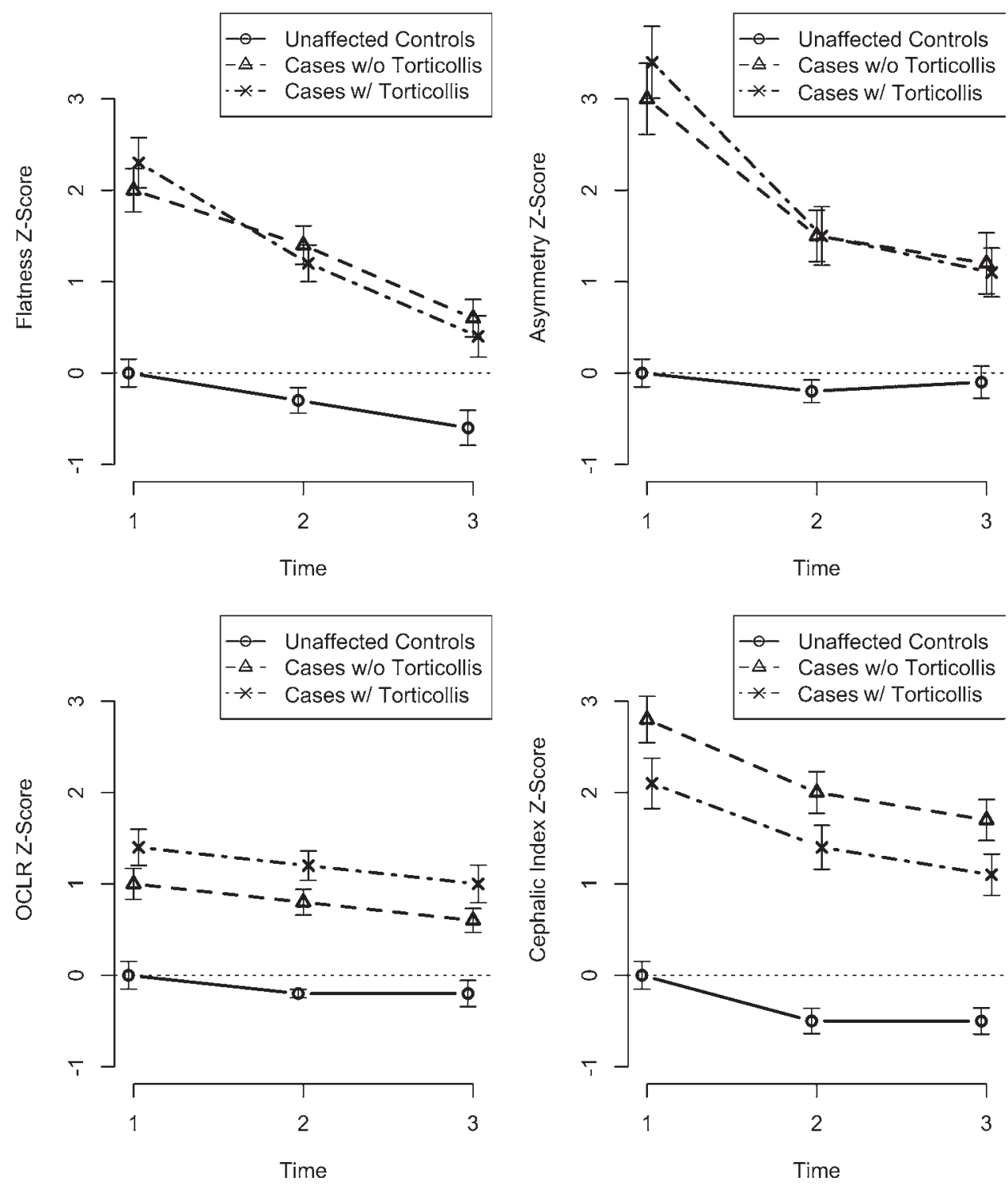

FIG. 4. Mean values and $95 \%$ confidence intervals of head shape measures over time for unaffected controls and patients with PPB (case group) with and without torticollis. Head shape data were available for one or more measures for 167 unaffected controls, 135 PPB patients without torticollis, and 100 PPB patients with torticollis at time 1. At time 2, one or more measures were available for 161 unaffected controls, 126 cases without torticollis, and 97 PPB patients with torticollis. At time 3, data were available for one or more measures for 151 unaffected controls, 112 cases without torticollis, and 92 PPB patients with torticollis.

ing showed a negligible advantage of helmet treatment in a sample of children with mild to moderate PPB (severe cases were excluded). ${ }^{14}$

We used the 95th percentile as an empirical clinical cutoff to determine persistent PPB, which would represent a score approximately 2 SDs above average. However, a score in this range does not necessarily indicate that deformation would be "noticeable" by others or that it would have a distinctly adverse effect on children's appearance. Treatments for PPB are sometimes advocated to prevent poor cosmetic outcomes, but we are unaware of any studies documenting the extent of deformation needed to produce clinically or socially meaningful differences. In previous studies of parent perceptions of head shape among children with a history of PPB, few parents were concerned about their child's appearance, although most continued to notice some degree of relative asymmetry. ${ }^{12}$ It seems unlikely that even the relatively large differences observed in this study would lead to measurable impact on social processes, but future research is needed to confirm this impression given the cost and potential liabilities of PPB treatment (e.g., child discomfort). Further, it is not clear whether persistent skull deformation has any implications for developmental outcomes.

The limitations of our study include the reliance on automated measures of head shape, which may be less precise in older children due to hair artifacts. Children wore close-fitting stocking caps during the collection of 3D imaging that helped to address this issue, though some remaining artifact is possible. Because this factor affected 

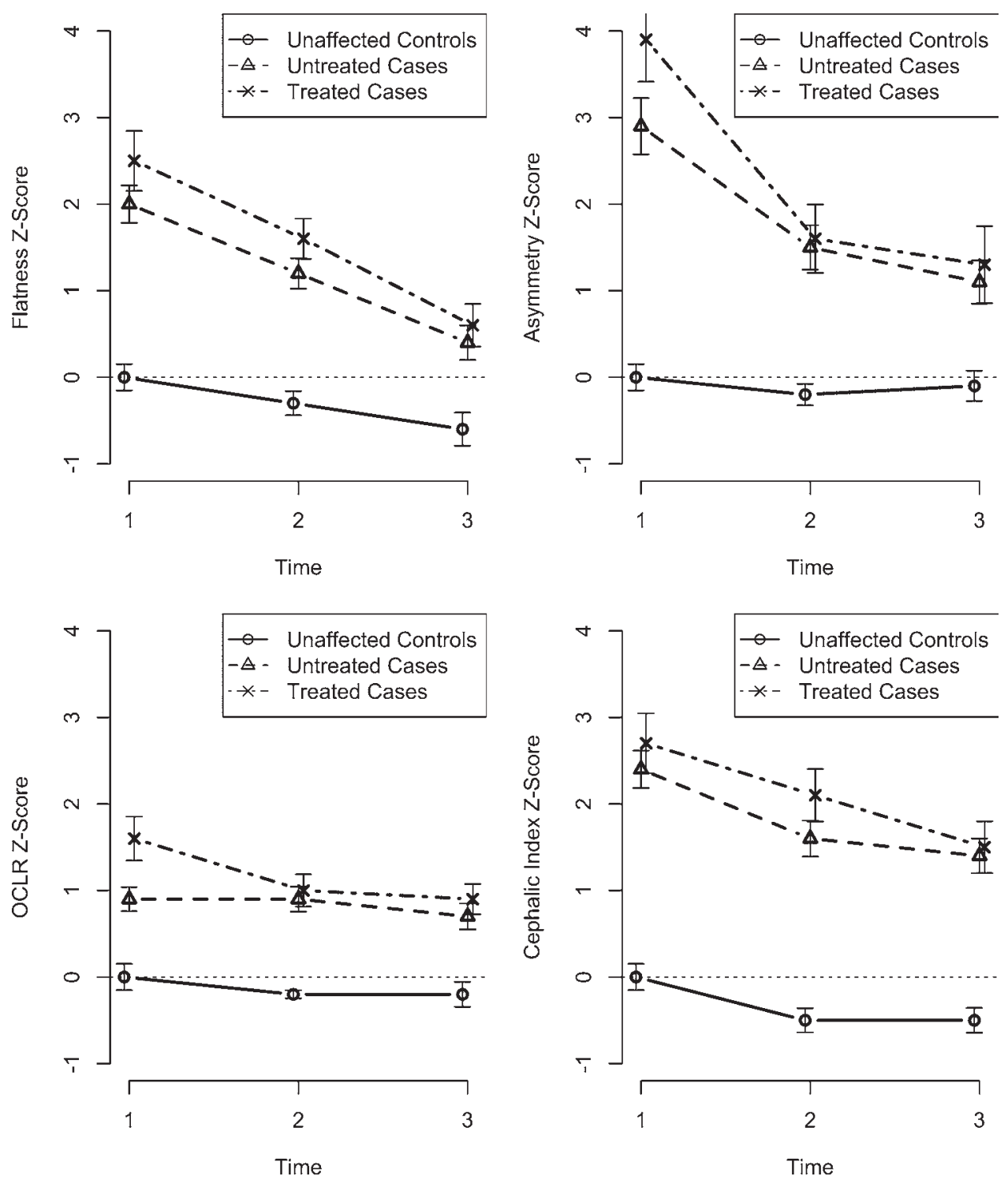

FIG. 5. Mean values and $95 \%$ confidence intervals of head shape measures over time for unaffected controls and cases by orthotic helmet or band treatment status. Head shape data were available for one or more measures for 167 unaffected controls, 165 untreated cases, and 63 treated cases (i.e., those who received a cranial orthosis and whose parents reported "adequate" or better compliance) at time 1. At time 2, one or more measures were available for 161 unaffected controls, 154 untreated cases, and 62 treated cases. At time 3, data were available for one or more measures for 151 unaffected controls, 143 untreated cases, and 54 treated cases.

both children with PPB and controls, such artifact would be expected to introduce greater error variance if anything, and would make it more difficult to detect group differences. Although we examined differences as a function of treatment history, our study was not designed to evaluate the efficacy of orthotic helmet treatment. As a result, there were baseline differences in head shape for treated versus untreated PPB patients. We also relied on parent-reported compliance with orthotic helmet treatment and we did not collect any other information that might have indicated the adequacy of helmet treatment. Further, we did not systematically collect data on repositioning and other strategies that might have attenuated the severity of deformation. Finally, our findings may have been affected to some extent by attrition, as there is evidence that more severely affected children were more likely lost to attrition.

\section{Conclusions}

Our findings suggest that deformation associated with PPB continues to improve to some extent until age 36 months. However, large differences in head shape remain between children with and without infancy PPB. Further, among children with PPB, cranial deformation persisted for those with and without a history of orthotic treatment history and for those with and without a history of physical or occupational therapy. Most children with PPB continue to show significant deformation on one or more measures 

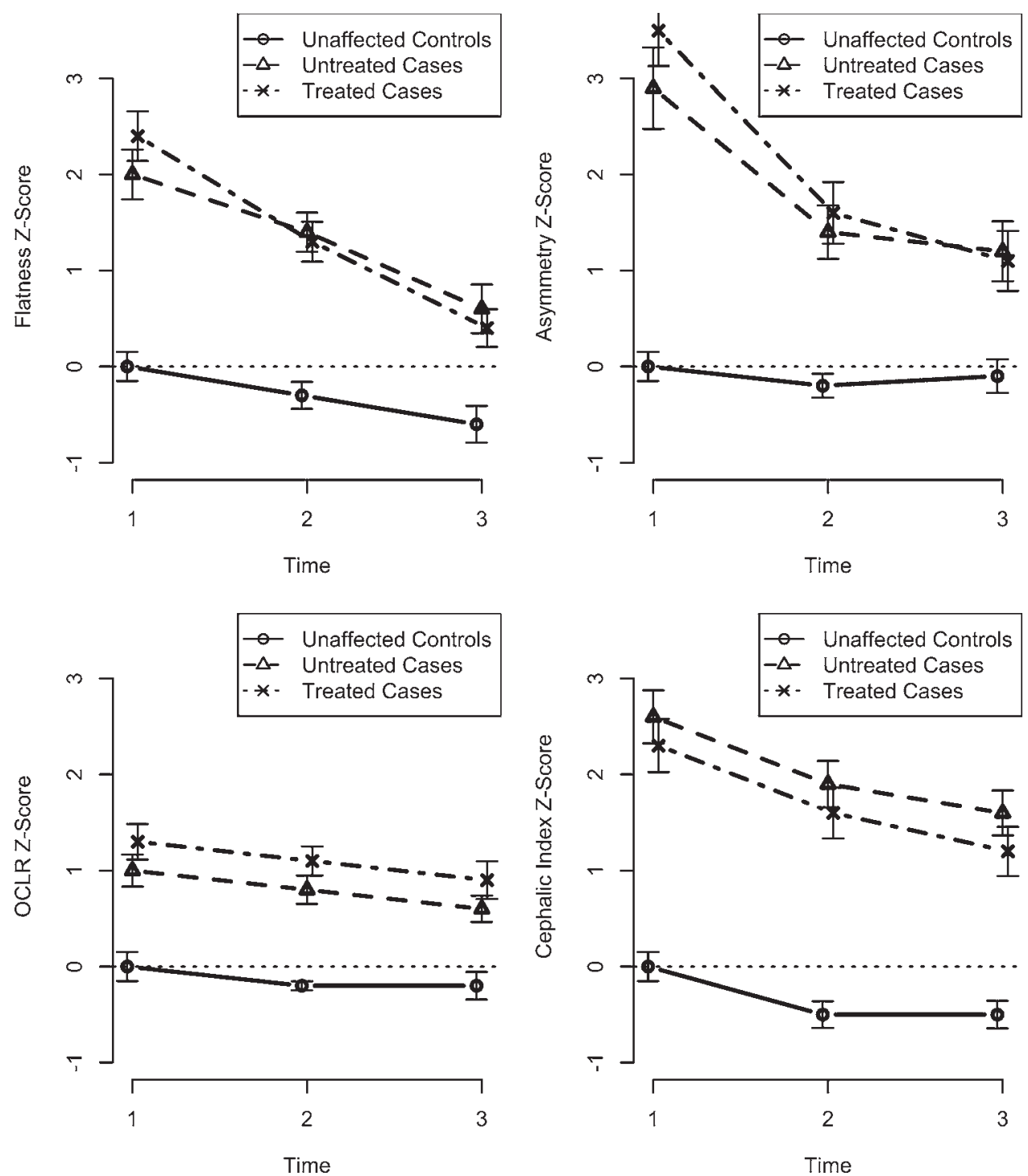

FIG. 6. Mean values and $95 \%$ confidence intervals of head shape measures over time for unaffected controls and cases by physical/occupational therapy treatment status. Time 1 head shape data were available for one or more measures for 167 unaffected controls, 116 untreated cases, and 109 treated cases (i.e., those who received physical or occupational therapy by time 3). At time 2 , one or more measures were available for 161 unaffected controls, 114 untreated cases, and 104 treated cases. At time 3, data were available for one or more measures for 151 unaffected controls, 104 untreated cases, and 96 treated cases.

of skull flattening and asymmetry. Additional research is needed to document the clinical and social implications of these differences and to explore factors associated with persistent PPB.

\section{Acknowledgments}

We thank Michael Cunningham, MD, PhD, and Carrie Heike, MD, MS, for completing clinician ratings of cranial deformation. We also thank Indriyati Atmosukarto, PhD, for developing our automated head shape measures, and Erik Stuhaug for his assistance with the collection of 3D images.

This work was supported by grants from the Eunice Kennedy National Institute of Child Health and Human Development (NICHD) to Dr. Speltz (R01 HD 046565) and Dr. Collett (R01 HD 080462).

\section{References}

1. Argenta LC, David LR, Wilson JA, Bell WO: An increase in infant cranial deformity with supine sleeping position. $\mathbf{J}$ Craniofac Surg 7:5-11, 1996

2. Atmosukarto I, Shapiro LG, Starr JR, Heike CL, Collett B, Cunningham ML, et al: 3D head shape quantification for infants with and without deformational plagiocephaly. Cleft Palate Craniofac J 47:368-377, 2010

3. Collett BR, Heike CL, Atmosukarto I, Starr JR, Cunningham ML, Speltz ML: Longitudinal, three-dimensional analysis of head shape in children with and without deformational plagiocephaly or brachycephaly. J Pediatr 160:673-678, 678.e1, 2012

4. Graham JM, Smith DW: Smith's Recognizable Patterns of Human Deformation. Philadelphia: Saunders/Elsevier, 2007

5. Hutchison BL, Hutchison LA, Thompson JM, Mitchell EA: 
Plagiocephaly and brachycephaly in the first two years of life: a prospective cohort study. Pediatrics 114:970-980, 2004

6. Hutchison BL, Stewart AW, Mitchell EA: Deformational plagiocephaly: a follow-up of head shape, parental concern and neurodevelopment at ages 3 and 4 years. Arch Dis Child 96:85-90, 2011

7. Kane AA, Mitchell LE, Craven KP, Marsh JL: Observations on a recent increase in plagiocephaly without synostosis. Pediatrics 97:877-885, 1996

8. Leppig KA, Werler MM, Cann CI, Cook CA, Holmes LB: Predictive value of minor anomalies. I. Association with major malformations. J Pediatr 110:531-537, 1987

9. McKinney CM, Cunningham ML, Holt VL, Leroux B, Starr JR: Characteristics of 2733 cases diagnosed with deformational plagiocephaly and changes in risk factors over time. Cleft Palate Craniofac J 45:208-216, 2008

10. Speltz ML, Collett BR, Stott-Miller M, Starr JR, Heike C, Wolfram-Aduan AM, et al: Case-control study of neurodevelopment in deformational plagiocephaly. Pediatrics 125:e537-e542, 2010

11. Steinberg JP, Rawlani R, Humphries LS, Rawlani V, Vicari FA: Effectiveness of conservative therapy and helmet therapy for positional cranial deformation. Plast Reconstr Surg 135:833-842, 2015

12. Steinbok P, Lam D, Singh S, Mortenson PA, Singhal A: Long-term outcome of infants with positional occipital plagiocephaly. Childs Nerv Syst 23:1275-1283, 2007

13. Turk AE, McCarthy JG, Thorne CH, Wisoff JH: The "back to sleep campaign" and deformational plagiocephaly: is there cause for concern? J Craniofac Surg 7:12-18, 1996
14. van Wijk RM, van Vlimmeren LA, Groothuis-Oudshoorn CG, Van der Ploeg CPB, IJzerman MJ, Boere-Boonekamp MM: Helmet therapy in infants with positional skull deformation: randomised controlled trial. BMJ 348:g2741, 2014

15. Xia JJ, Kennedy KA, Teichgraeber JF, Wu KQ, Baumgartner JB, Gateno J: Nonsurgical treatment of deformational plagiocephaly: a systematic review. Arch Pediatr Adolesc Med 162:719-727, 2008

\section{Disclosures}

The authors report no conflict of interest concerning the materials or methods used in this study or the findings specified in this paper.

\section{Author Contributions}

Conception and design: Collett, Speltz. Acquisition of data: Collett, Speltz. Analysis and interpretation of data: Collett, Leroux, Wallace, Shao, Speltz. Drafting the article: Collett. Critically revising the article: Collett, Wallace, Gallagher, Speltz. Reviewed submitted version of manuscript: all authors. Approved the final version of the manuscript on behalf of all authors: Collett. Statistical analysis: Leroux, Wallace, Shao. Study supervision: Collett, Speltz.

\section{Correspondence}

Brent Collett: Seattle Children's Research Institute, Seattle, WA. bcollett@u.washington.edu. 